\title{
ÍNDICES DE DEPRESSÃO DE MULHERES COM CÂNCER DE COLO UTERINO EM TRATAMENTO, EM UM HOSPITAL DE REFERÊNCIA NO BAIXO AMAZONAS, PARÁ.
}

\author{
DEPRESSION INDEXES OF WOMEN WITH CERVIX CANCER IN TREATMENT, \\ IN A REFERRAL HOSPITAL IN THE LOWER AMAZON, PARÁ. \\ Brenda Nunes Corrêa ${ }^{1}$, Gabriela Noronha Fortes ${ }^{2}$, Patrícia Mineiro de Oliveira ${ }^{2}$ \\ Especialista em oncologia- Universidade do Estado do Pará ${ }^{1}$. \\ Psicóloga. Doutoranda em Ciências pela Universidade de São Paulo (USP). Universidade do Estado do Pará ${ }^{2}$
}

Abstract

The objective was to analyze the analysis of the depression indexes of patients with cervical cancer during treatment, in a reference hospital in the region of Lower Amazon, Pará. This is a descriptive study, a quantitative approach, whose sampling was characterized as non-probabilistic for convenience. Fifteen women participated, diagnosed with cervical cancer undergoing treatment. The instruments used were a sociodemographic and clinical questionnaire, Beck's Depression Inventory and the Mini Mental State Examination. The data were through descriptive statistics. Most of the participants were over 50 years old, with incomplete elementary education, monthly income below the minimum wage and in a stable union. It was observed that the majority of participants, $80 \%$, had a depressive state between levels of severe level during treatment for cervical cancer, with the highest recurrence rate at moderate intensity, which corresponded to $46.7 \%$. As for cognitive status, $80 \%$ of women had a normal response and $20 \%$ of women with an altered response. Most participants in cancer treatment for CCU dissipate depressive state, 3 of which, with changes in mental status, evident cognitive decline. Therefore, it is necessary to know and evaluate depressive symptoms, valuing the subjectivity and individuality of women in illness and treatment of cervical cancer in an ethical and professional manner.

Keywords: cervical cancer, depression, integral attention to women's health.
Resumo

Objetivou-se analisar os índices de depressão das pacientes com câncer de colo uterino durante o tratamento, em um hospital de referência na região do Baixo Amazonas, Pará. Trata-se de um estudo descritivo, abordagem quantitativa, cuja amostragem foi caracterizada como não probabilística por conveniência. Participaram 15 mulheres, com diagnóstico de câncer de colo uterino em tratamento. Os instrumentos utilizados foram um questionário sociodemográfico e clínico, Inventário de Depressão de Beck e o Mini Exame do Estado Mental. Os dados foram analisados através da estatística descritiva. A maioria das participantes tinha idade acima de 50 anos, com ensino fundamental incompleto, renda mensal abaixo de um salário mínimo e em uma união estável. Observou-se que a maioria das participantes, $80 \%$, apresentou estado depressivo entre os níveis leve a grave durante o tratamento para câncer cervical, tendo o maior índice de recorrência na intensidade moderada que correspondeu a 46,7\%. Quanto ao estado cognitivo, $80 \%$ das mulheres apresentaram resposta normal e $20 \%$ das mulheres com resposta alterada. A maioria das participantes em tratamento oncológico por câncer de colo uterino apresentaram estado depressivo, sendo que 3 destas, com alteração no estado mental, indicando possível declínio cognitivo. Portanto, é necessário conhecer $e$ avaliar os sintomas depressivos, valorizando a subjetividade $e$ individualidade das mulheres em adoecimento $e$ tratamento de câncer do colo uterino de maneira ética e profissional.

Palavras-chave: câncer de colo uterino, depressão, atenção integral à saúde da mulher. 


\section{Introdução}

Na saúde da população feminina, o câncer de colo uterino (CCU), também chamado de câncer cervical, é um dos de maior incidência e mortalidade, com implicações nas áreas afetivas, de autoimagem, sociais, sexuais e psicológicas das mulheres acometidas ${ }^{1}$. A literatura aponta algumas variáveis e riscos relacionados ao aparecimento desse tipo de câncer nas mulheres, se destacam a infecção persistente após exposição a alguns subtipos de alto risco do Papilomavírus Humano (HPV) (HPV 16 e 18), a multiparidade, início precoce da atividade sexual, uso prolongado de contraceptivos orais (estrogênio), tabagismo ou exposição ao ambiente do tabaco e a imunossupressão ${ }^{2-4}$

O Instituto Nacional de Câncer- INCA ${ }^{5}$ destaca que em âmbito mundial, o câncer de colo uterino, ocupa o sétimo lugar sendo o quarto tipo mais comum entre as mulheres e a neoplasia do aparelho reprodutor feminino mais frequente nos países em desenvolvimento. Para cada ano do triênio 2020-2022, são esperados para o Brasil cerca de 16.590 casos novos de câncer do colo uterino, com risco estimado de 15,43 casos a cada 100 mil mulheres ${ }^{5}$. Na Região Norte, a incidência do câncer do colo do útero tem impacto de relevância por ser a única região do país onde as taxas de câncer de mama (1.970) e colo do útero (1.940) se equivalem entre as mulheres, ocupando o segundo lugar entre os tumores mais incidentes de localização primária, assim como para o estado do Pará, que foram estimados para o ano de 2020780 casos novos ${ }^{5}$.

Quanto ao número de óbitos por CCU em âmbito nacional ocupou a quarta posição, sendo encontrado na região norte as maiores taxas de mortalidade do país e o único lugar com nítida tendência temporal de crescimento ${ }^{6}$. As manifestações clínicas ocorrem de forma gradativa correlacionados à história natural de progressão da doença. Entre as queixas podem estar o sangramento intermitente durante as relações sexuais. Nos casos avançados, pode cursar com dores abdominais associadas a queixas intestinais e urinárias ${ }^{2,4,7}$. A neoplasia do colo do útero, apesar de prevenível, se mantém como o grupo de tumores mais comum entre as mulheres, tendo em vista as falhas nos diferentes níveis de atenção à saúde da população ${ }^{8}$.

O tratamento efetivo para o câncer cervical depende do estadiamento do tumor e de fatores psicossociais, onde diferentes modalidades de condutas terapêuticas podem ser empregadas, como a cirurgia, quimioterapia, radioterapia externa e braquiterapia ${ }^{1}$. Vários fatores contribuem para alterações na qualidade de vida das mulheres com câncer ginecológico. Dentre elas, destacam-se as alterações funcionais decorrentes do tratamento, como as cirurgias e a radioterapia que pode danificar a musculatura pélvica, as raízes nervosas sacrais e a mucosa vaginal. Além dessas, os efeitos colaterais da quimioterapia, como a diarreia, náusea, vômito pode ter repercussão no peso bem como ter alterações hormonais. Tudo isso está intimamente relacionado a fatores psicológicos de crenças quanto sobre a origem do câncer, mudanças na autoimagem, percepção corporal, autoestima baixa, conflitos familiares, medos e preocupações $^{9}$.

Nesse processo, é importante atentar para a capacidade de ajustamento emocional da pessoa em tratamento oncológico com relação a seu adoecer e tratamento, pois, frequentemente se observam sentimentos de tristeza, pesar e sintomas depressivos ${ }^{9}$ nessa população. De acordo com a Organização Mundial da Saúde ${ }^{10}$, a depressão é um transtorno mental caracterizado como leve, moderado ou grave que dependerá da intensidade dos sintomas, sendo um problema médico e um grande problema de saúde pública. Conforme o Manual de diagnóstico e estatístico de transtornos mentais $^{11}$, o diagnóstico de depressão ocorre quando presentes pelo menos cinco de um total de nove sintomas, se mostrando presentes um ou dois dos principais sintomas, como o humor deprimido e a anedonia (incapacidade de se sentir alegre). Os demais sintomas são referentes a sentimentos de: inutilidade/culpa, insônia ou hipersonia, ganho ou perda de peso, agitação ou retardo psicomotor, fadiga/perda de energia, baixa concentração e ideação suicida ${ }^{11}$.

Os fatores que contribuem para o estado de humor deprimido do paciente oncológico em tratamento podem ser o convívio com a dor, questões relacionadas à autoimagem (percepção corporal), funcionalidade sexual e dependência do outro. Além disso, o isolamento, preconceitos, processos de separação e questões relacionadas à morte e ao morrer, que envolvem a própria terminalidade, além de suportar os efeitos colaterais dos tratamentos, os inúmeros retornos ao hospital de referência com episódios de internação, questão socioeconômica e vulnerabilidade social e mudanças no âmbito familiar $^{1}, 12,13,14,15$. Dessa forma, tanto 0 momento do diagnóstico quanto de tratamento, geram mudanças físicas e psicológicas que podem afetar a vida das mulheres em diversos níveis e contextos, sendo fundamental atentar 
para a capacidade de ajustamento emocional da pessoa em tratamento oncológico em relação ao seu adoecer e tratamento ${ }^{1,12,16}$.

Diante da importância clínica e epidemiológica da neoplasia no colo uterino ${ }^{3,5}$ na região Norte, associado ao fato de haver poucos achados na literatura este trabalho visa analisar os índices de depressão das pacientes com câncer de colo uterino durante o tratamento, em um hospital de referência na região do Baixo Amazonas no Pará.

\section{Metodologia}

Trata-se um estudo descritivo do tipo quantitativo, com corte transversal.

A pesquisa foi realizada no Hospital Regional do Baixo Amazonas do Pará Dr. Waldemar Penna, Oeste do Pará, tendo o ambulatório de radioterapia como local da coleta de dados. Localizado na 9 a regional de saúde, o local oferta atendimento de média e alta complexidade à população $100 \%$ referenciado pelo Sistema Único de Saúde (SUS), sendo referência aos pacientes oncológicos do município de Santarém e referência no Norte do Brasil para residentes em 20 municípios da região oeste do Pará.

A amostra se caracteriza como não probabilística por conveniência ${ }^{17}$ tendo como participantes 15 mulheres. As participantes estavam em vigência de tratamento oncológico ambulatorial no setor de radioterapia do hospital, nos meses de julho à novembro de 2020, e concordaram assinando o Termo de Consentimento Livre e Esclarecido (TCLE).

Os critérios de inclusão foram: mulheres com idade acima de 18 anos, com diagnóstico de câncer do colo do útero, em tratamento mínimo três sessões de radioterapia realizadas no período da coleta de dados. Como critérios de exclusão estavam mulheres com diagnóstico de transtorno psicótico, deficiência intelectual grave ou demências detectadas em exame clínico anterior a coleta de dados. As pacientes foram convidadas a participar da pesquisa, e concordaram assinando o Termo de Consentimento Livre e Esclarecido (TCLE). Nesta pesquisa houve uma recusa, uma desistência e três não se enquadraram nos critérios de inclusão.

Após assinatura do TCLE, as participantes foram individualmente a uma sala reservada dentro da unidade de atuação, com iluminação e materiais avaliativos adequados, respeitando as orientações da $\mathrm{OMS}^{18}$ devido o panorama mundial de pandemia da Covid 19, com distanciamento mínimo de 1 a 2 metros entre participante e pesquisadora, sendo oferecidas máscaras cirúrgicas, álcool gel e pia com água e sabão para higienização das mãos. 0 sigilo foi preservado através da enumeração dos instrumentais das participantes (ex: P1, P2, P3).

Os instrumentos utilizados foram um questionário sociodemográfico e clínico, elaborado pela pesquisadora, a fim de caracterização das participantes com as variáveis relacionadas à: idade, estado civil, escolaridade, ocupação, procedência, paridade (no de filhos), renda, religião e dados clínicos, coletados a partir do prontuário físico ou digital, ocorrido após assinatura do Termo de Compromisso para a Utilização de Dados dos Prontuários dos Pacientes (TCUD). O Mini Exame do Estado Mental (MEEM) ${ }^{19}$ foi um instrumento utilizado, composto por questões agrupadas em sete categorias, cada uma delas com o objetivo de avaliar um grupo de funções cognitivas específicas, com pontuação total podendo variar de zero até o máximo de 30 pontos. Foram utilizados os pontos de corte que consideram a estratificação por níveis ou anos de escolaridade $^{19}$. As pontuações indicativas de declínio cognitivo foram: (a) 13 pontos para analfabetos; (b) 18 pontos para indivíduos com baixa ou média escolaridade; e (c) 26 pontos para aqueles com alto nível de escolarização.

Também foi aplicado o Inventário de Depressão de Beck $\mathrm{BDI}^{20}$ para identificação dos índices de depressão a partir dos graus crescentes de intensidade de sintomas depressivos. Os vinte e um itens avaliados pelo BDI são: tristeza, pessimismo, sensação de fracasso, insatisfação, sensação de culpa, expectativa de punição, autodepreciação, autocrítica, ideias suicidas, crise de choro, irritabilidade, retração social, indecisão, distorção da imagem corporal, inibição para o trabalho, distúrbios do sono, fadiga, perda de apetite, perda de peso, preocupação somática e diminuição da libido ${ }^{20}$. Com escore de zero a três e quatro alternativas, a soma dos escores dos itens individuais fornece um escore total, onde o maior é 63, que indica alto grau de depressão e o mais baixo é o zero, que corresponde à ausência de depressão. Os scores de zero a 11 são sem depressão ou depressão mínima; escores de 12 a 19, depressão leve; escores de 20 a 35 , depressão moderada; escores de 36 a 63 depressão grave.

O estudo seguiu com os aspectos éticos de pesquisa e estudo com seres humanos regidos pela Resolução no 466/2012 do Conselho Nacional de Saúde ${ }^{21}$. Após a aprovação pela 
direção do Hospital referência e do Comitê de Ética e Pesquisa (CEP) da Universidade do Estado do Pará, aprovado com o CAAE 32446120.5.0000.5168, com a assinatura do TCLE.

A análise estatística foi descritiva em número absoluto e percentuais, médias e desvio padrão utilizando o programa Microsoft Excel 2010.

\section{Resultados e Discussão}

O perfil sociodemográfico das participantes do estudo foi composto por 15 mulheres, com idade mínima de 32 anos e máxima de 82 anos (Média: 50,4; DP $\pm 11,77$ ), onde a maioria apresentou idade média a partir de 50 anos, tendo predominância de mulheres na faixa etária de 50-59 anos 9 (66,63\%), seguido de $32-40$ anos $(26,7 \%)$ e $80-82$ anos $(6,7 \%)$ (Tabela 1). A média de idade corrobora com os achados em três estudos distintos com mulheres em tratamento para CCU realizados no centro de referência no Rio de Janeiro ${ }^{15}$, em um hospital de Palmas-TO ${ }^{22}$, e na cidade de Macapá/AP ${ }^{23}$. Divergindo de outros, onde a predominância da idade média foi a partir dos 40 anos ${ }^{13,24}$.

Os resultados concordam com os estudos mencionados ${ }^{15,22,23}$ e com os dados fornecidos pelo INCA ${ }^{5}$, que apontam incidências elevadas de câncer ginecológico em mulheres com idades acima dos 40 anos de idade, sendo importantes os programas de rastreamento e diagnóstico precoce quanto aos exames citopatológicos. No Brasil, o SUS recomenda que se inicie o rastreamento para o câncer do colo do útero aos 25 anos de idade para mulheres que já iniciaram a atividade sexual - e se encerre aos 64 anos, desde que as mulheres sem história prévia de doença préinvasora tenham dois exames citopatológicos negativos consecutivos nos últimos cinco anos. Os dois primeiros exames devem ser realizados com intervalo anual e, se ambos os resultados forem negativos, os próximos devem ser realizados a cada três anos ${ }^{2}$.

Apesar das altas taxas de cura quando o câncer de colo uterino é detectado precocemente, muitas mulheres não realizam o exame ginecológico periodicamente devido fatores subjetivos e sócio-culturais-ambientais ${ }^{7}$. A ineficiência de informações sobre a existência dos procedimentos de rastreamento, ausência de patologia genital ou de prescrição médica e dificuldade de acesso aos serviços de saúde no país são fatores relevantes nessa discussão ${ }^{7}$.

A seguir, na tabela 1 serão apresentadas as características máximas e mínimas das participantes do estudo.

Tabela 1: Características sociodemográficas das participantes com CCU.

\begin{tabular}{lc}
\hline CARACTERÍSTICAS & $\mathbf{N}(\%)$ \\
\hline Idade & $9(66,63)$ \\
$50-59$ & $1(6,7)$ \\
80 a 82 & $7(46,7)$ \\
\hline Estado Civil & $2(13,3)$ \\
União Estável & \\
Divorciada & $7(46,7)$ \\
\hline Escolaridade & $1(6,7)$ \\
Ens. Fund. Inc. & $7(46,7)$ \\
Analfabeta & $2(13,3)$ \\
\hline Renda familiar & \\
Menos de 1 salário mínimo & $8(53,3)$ \\
1 salário mínimo & $7(46,7)$ \\
\hline Religião & \\
Católica & \\
Evangélica & \\
\hline Ocupação & $5(33,3)$ \\
Do lar & $1(6,7)$ \\
Aposentada & \\
\hline No de filhos & $5(33,3)$ \\
3 a 4 & $2(13,3)$ \\
5 e sem filhos & \\
\hline
\end{tabular}

Fonte: Dados da pesquisa.

COM CÂNCER DE COLO UTERINO EM TRATAMENTO, EM UM HOSPITAL DE REFERÊNCIA NO BAIXO AMAZONAS, PARÁ. Rev. Saúde.Com 2021; 17(2):2157-2169. 
Em relação à procedência das participantes, se observou que a maioria (10) delas precisaram se deslocar das cidades próximas e até uma mulher do município de Macapá ( $A P)$, para conseguir iniciar o tratamento indicado, fato este, que confirma o local no município como referência para atendimento oncológico na região Oeste do Pará. Das oriundas do município de Santarém (PA) foram 33,32 \%, seguido por $13,32 \%$ de Oriximiná (PA) e 6,7 \% dos demais municípios, cada uma, correspondendo aos municípios de Jacareacanga (PA), Alenquer (PA), Prainha (PA), Itaituba (PA), Monte Alegre (PA), Curuai (PA), São Joaquim do Tapará (PA).

Quanto ao estado civil, à maioria das participantes (10) tem um parceiro, destas, $46,7 \%$ encontrava-se em união estável e $20 \%$ são casadas. Das outras participantes (5), 20\% eram solteiras e $13,3 \%$ divorciadas. Os dados corroboraram aos achados da autora ${ }^{24}$ quanto a predominância da união estável entre as participantes, fato este, que diverge de alguns estudos apontaram o estado civil casado como mais predominante entre as mulheres com $\mathrm{CCU}^{22,23,25,26}$.

Nos níveis de escolaridade, verificou-se que a maioria das mulheres $(46,7 \%)$, possuía até o ensino fundamental incompleto, corroborando com a literatura $15,22,25,26$. Com ensino fundamental completo/ensino médio incompleto foram $6,7 \%$, com ensino médio completo/ ensino superior completo, $13,3 \%$ e somente uma participante referiu não ser alfabetizada. A escolaridade é fator importante no que se trata ao planejamento e à implementação de atividades educativas, devendo ser inclusivas e de fácil compreensão e participação das mulheres com câncer nas orientações propostas.

Com relação à renda, a maioria das participantes, $46,7 \%$ se mantém com menos de um salário mínimo, 40\% com mais de um salário e $13,3 \%$ com apenas um salário mínimo. Autores apontam que o menor nível socioeconômico implica em maiores taxas de não realização do exame de Papanicolau, consequentemente levando a um aumento do risco de desenvolvimento do câncer cervical ${ }^{25}$.

Os resultados com relação à ocupação apontaram que a maioria se dedica às atividades do lar $(33,3 \%)$ concordando com os achados na literatura $^{15,22}$ divergindo de outros, em que a maior parte foram mulheres aposentadas e domésticas $^{22,}{ }^{23}$. As demais se declararam agricultoras e professoras (20\%), vendedoras $(13,3 \%)$, auxiliar de serviços gerais e aposentadas $(6,7 \%)$. No que se refere à quantidade de filhos, a maioria tem de 3 a 4 filhos $(33,3 \%)$ e mediana do número de filhos de três, concordando com os achados na literatura $^{15}$. As mulheres com 5 filhos corresponderam a $20 \%$, seguido 1 a 2 filhos e acima de cinco filhos com 13,3\%, respectivamente. $\mathrm{Em}$ um estudo ${ }^{23}$ realizado mostrou que $100 \%$ das participantes tinham filhos, fato divergente dos resultados desta pesquisa, em que $20 \%$ das mulheres não tem filhos.

A característica religiosa foi presente em todas as participantes, sendo a maioria $(53,3 \%)$ católica e $(46,7 \%)$ evangélica, corroborando com os achados na literatura ${ }^{12,22}$. Diante do acometimento pelo câncer, a literatura apontou que a religiosidade e a espiritualidade podem ser um instrumento de suporte e conforto para auxiliar no enfrentamento das adversidades causadas pelo câncer e por seu tratamento ${ }^{12}$.

Embora o câncer de colo do útero possa acometer mulheres, independente da faixa etária, perfil socioeconômico, escolaridade, região demográfica e espiritualidade, faz-se necessário destacar que o nível educacional e sócio econômico são características marcantes do CCU, por serem nesses grupos que se encontram maiores dificuldades de acessibilidade para deteç̧ão e tratamento precoce da neoplasia, perpassando dificuldades econômicas, geográficas, problemas estruturais, questões culturais, religiosas, tabus e preconceito por parte dos parceiros ${ }^{1,12}$.

Outro ponto relevante são as mudanças na rotina e estilo de vida, ocorridas durante o tratamento oncológico que podem gerar estresse e preocupação ${ }^{1,12-15}$. Um $\operatorname{artigo~}^{16}$ de revisão destaca possíveis fatores estressores para mulheres com CCU, como o momento a partir do aparecimento dos primeiros sinais e sintomas da doença; o crescimento do tumor; à medida que o diagnóstico se apresenta e o tratamento recomendado. Associado a isso, ainda existem os problemas familiares, tais como a violência severa por parte do parceiro e dentre outros ${ }^{16}$.

Neste estudo, os resultados quanto às características do adoecimento e tratamento oncológico para CCU realizado, demonstrou que a maioria das participantes $(60 \%)$ realizava radioterapia concomitante à quimioterapia corroborando com a literatura ${ }^{14,15}$. Mais da metade das participantes, 13 (53,33\%) apresentavam ocorrência de metástase (processo de disseminação do câncer no organismo), 20\% realizavam tratamento devido recidiva (reaparecimento do câncer). Quanto ao estadiamento da doença, a maioria (12) encontrava-se entre os estádios III a IV. Das que 
relataram realizar tratamento/acompanhamento em saúde mental (psicologia e/ou psiquiatria) prévio e/ou concomitante ao tratamento relacionado à depressão, apenas 2 (13,33\%), conforme demonstrado na tabela 2 .

Tabela 2. Características quanto o adoecimento e tratamento oncológico.

\begin{tabular}{lc}
\hline \multicolumn{1}{c}{ CARACTERíSTICAS } & N (\%) \\
\hline $\begin{array}{l}\text { Tratamentos realizados } \\
\text { Procedimento cirúrgico }{ }^{+} \\
\text {radioterapia concomitante à } \\
\text { quimioterapia }\end{array}$ & $5(33,33)$ \\
$\begin{array}{l}\text { Radioterapia exclusiva } \\
\text { Radioterapia concomitante à } \\
\text { quimioterapia }\end{array}$ & $1(6,67)$ \\
\hline Recidiva & $9(60)$ \\
Sim & \\
Não & $3(20)$ \\
\hline Metástase & $12(80)$ \\
Sim & \\
Não & $8(53,33)$ \\
\hline Acompanhamento/tratamento em & $7(46,67)$ \\
saúde mental & \\
Sim & $2(13,33)$ \\
Não & $13(86,67)$ \\
\hline Fonte: Dados da pesquisa. &
\end{tabular}

Ao lidar com diagnóstico de câncer, os impactos emocionais podem ser potencializados por acumular um misto de sensações fortalecidas com a realização do tratamento oncológico (cirurgia, quimio e radioterapia) ${ }^{1,12,14,15}$. O diagnóstico e o respectivo tratamento trazem diversas consequências para a vida das pacientes a nível social, físico, sexual e psicológico. Estas consequências psicológicas advindas do diagnóstico e do tratamento para o CCU levam a uma redução da qualidade de vida. Os resultados corroboram com a literatura ao mostrar que o tratamento para o câncer ginecológico frequentemente envolve o uso de radioterapia e quimioterapia, como evidenciado neste estudo.

Os resultados encontrados apresentaram que mais da metade das participantes $(66,67 \%)$ precisaram se deslocar de seu município para iniciar o tratamento indicado, possuem renda inferior a um salário mínimo, escolaridade predominante no ensino fundamental incompleto, corroborando com os autores que destacam elevado sofrimento psíquico devido a distância do núcleo familiar pelas mulheres que residem em outra localidade do tratamento, condições socioeconômicas, dependência de acompanhantes, necessidade de suporte, sentimentos de impotência e vergonha, mudanças nas suas atividades diárias, e se em tratamento de radioterapia, os efeitos adversos na pele que se caracterizam por radiodermatites variando de um leve eritema e prurido, descamação seca ou úmida à necrose tecidual, fadiga, diarreia, náusea, incontinência urinária, linfedema, estenose vaginal, falta de lubrificação vaginal, dispareunia, distúrbios do sono, atividade sexual prejudicada e pensamentos de morte/finitude, estresse e depressão ${ }^{1,8,13-16}$.

No que se refere à morbidade psicológica no câncer, a literatura ${ }^{27}$ aponta que o alto índice de sofrimento emocional em pacientes oncológicos faz parte de um processo de ajustamento, apontando a depressão como o transtorno mais frequente no adoecimento com câncer quando comparado com pacientes que apresentam outros tipos de doenças, ou ainda a prevalência de transtorno depressivo em pessoas com diagnóstico oncológico do que aquela encontrada na população geral, variando a partir da escolha do tamanho amostral e instrumentais selecionados para rastreio ${ }^{9}$.

Analisando a intensidade de sintomas depressivos das mulheres com CCU desta pesquisa, $80 \%$ (12 mulheres), apresentavam estados depressivos em níveis diferentes que variaram entre leve, moderado a grave (Figura 1). No BDI, as mulheres apresentaram pontuação mínima de 03 pontos e a máxima de 45 pontos, com uma média de $23,6( \pm 12,5)$ pontos. 0 maior índice de depressão entre as mulheres deste estudo se deu na intensidade moderada, correspondendo a 46,7\%, seguida pela mínima e grave com $20 \%$, respectivamente. O nível leve foi o de menor ocorrência, com 13,3\%, conforme exposto na figura 1. 
Figura 1: Intensidade da depressão nas participantes-BDI

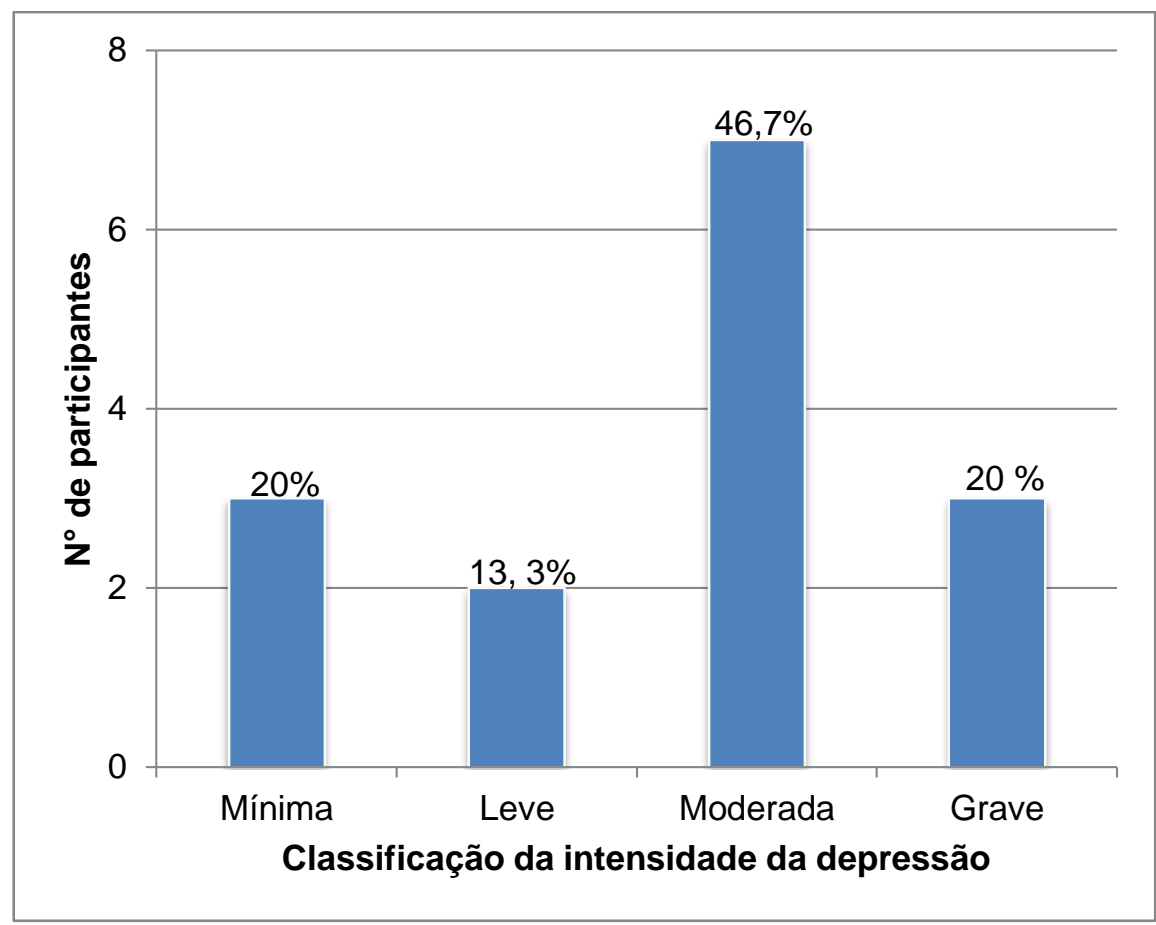

Os resultados apontaram que o estado depressivo ocorreu entre a maioria das participantes, corroborando com a literatura quanto aos elevados índices de humor deprimido nas pessoas em adoecimento e tratamento por câncer de colo uterino ${ }^{14,15}$. Foi encontrado um estudo ${ }^{13}$ que apresentou resultados diferentes no se refere ao nível de depressão em mulheres com CCU, ao demonstrar que a maior parte das participantes apresentava níveis mínimos ou leves, fato este, divergente aos dados deste estudo.

Autores destacam que os índices de depressão tendem a aumentar de maneira considerável de acordo com o agravamento do câncer, presença de dor, metástases, recidivas, gerando impactos sobre a qualidade de vida e evolução do adoecimento, sendo importante o conhecimento de tais características no cuidado a esse público ${ }^{28}$. No estudo ${ }^{13}$ que investigou a comparação das percepções da doença e os indicadores de ansiedade e depressão em mulheres com câncer cervical e mulheres com câncer de mama, concluiu que todas as mulheres apresentaram nível de ansiedade de moderado a grave. Contudo, para as participantes com câncer cervical, a doença teve uma maior conotação de sofrimento psicológico.
A respeito dos dados sintomatológicos do $\mathrm{BDI}$ com relação à depressão, a maioria das

participantes referiu à perda de libido, presente em 93,3\% (14) das mulheres seguidas pela perda de peso, preocupações somáticas, insônia, choro, dificuldades para trabalhar, perda de apetite, fadiga e irritabilidade como sintomas mais recorrentes entre as mulheres do estudo em tratamento oncológico (Tabela 3). 0 sentimento de insatisfação correspondeu a 9 (60\%) das participantes.

O pessimismo, dificuldade de tomada de decisão e mudança na autoimagem corresponderam a 8 (53,33\%). O percentual sintomatológico de menor recorrência se referiu ao sentimento de culpa, em que $80 \%$ das mulheres o negaram como presente. A ideação suicida apareceu no estudo com 4 (26, 67\%), corroborando aos achados no estudo que avaliou a depressão e comportamento suicida em pacientes oncológicos assim como a prevalência e fatores associados ${ }^{28}$. Na tabela 3 será apresentada a ocorrência dos sintomas de depressão entre as participantes. 
Tabela 3: Sintomas de depressão das participantes a partir do BDI.

\begin{tabular}{|c|c|}
\hline SINTOMAS & $\mathbf{N}(\%)$ \\
\hline \multicolumn{2}{|l|}{ Tristeza } \\
\hline Sim & $5(33,33)$ \\
\hline Não & $10(66,67)$ \\
\hline \multicolumn{2}{|l|}{ Pessimismo } \\
\hline Sim & $8(53,33)$ \\
\hline Não & $7(46,67)$ \\
\hline \multicolumn{2}{|c|}{ Sentimento de fracasso } \\
\hline Sim & $5(33,33)$ \\
\hline Não & $10(66,67)$ \\
\hline \multicolumn{2}{|l|}{ Insatisfação } \\
\hline Sim & $9(60)$ \\
\hline Não & $5(40)$ \\
\hline \multicolumn{2}{|l|}{ Culpa } \\
\hline Sim & $3(20)$ \\
\hline Não & $12(80)$ \\
\hline \multicolumn{2}{|l|}{ Punição } \\
\hline Sim & $4(26,67)$ \\
\hline Não & $11(73,33)$ \\
\hline \multicolumn{2}{|c|}{ Autoaversão } \\
\hline Sim & $5(33,33)$ \\
\hline Não & $10(66,67)$ \\
\hline \multicolumn{2}{|c|}{ Autoacusações } \\
\hline Sim & $6(40)$ \\
\hline Não & $9(60)$ \\
\hline \multicolumn{2}{|c|}{ Ideação suicida } \\
\hline Sim & $4(26,67)$ \\
\hline Não & $11(73,33)$ \\
\hline \multicolumn{2}{|l|}{ Choro } \\
\hline Sim & $12(80)$ \\
\hline Não & $3(20)$ \\
\hline \multicolumn{2}{|c|}{ Irritabilidade } \\
\hline Sim & $10(66,67)$ \\
\hline Não & $5(33,33)$ \\
\hline \multicolumn{2}{|c|}{ Retraimento social } \\
\hline Sim & $4(26,67)$ \\
\hline Não & $11(73,33)$ \\
\hline \multicolumn{2}{|c|}{ Dificuldade de tomada de decisão } \\
\hline Sim & $8(53,33)$ \\
\hline Não & $7(46,67)$ \\
\hline \multicolumn{2}{|c|}{ Mudança na autoimagem } \\
\hline Sim & $8(53,33)$ \\
\hline Não & $7(46,67)$ \\
\hline \multicolumn{2}{|c|}{ Dificuldade para trabalhar } \\
\hline Sim & $11(73,33)$ \\
\hline Não & $4(26,67)$ \\
\hline
\end{tabular}

ÍNDICES DE DEPRESSÃO DE MULHERES COM CÂNCER DE COLO UTERINO EM TRATAMENTO, EM UM HOSPITAL DE REFERÊNCIA NO 


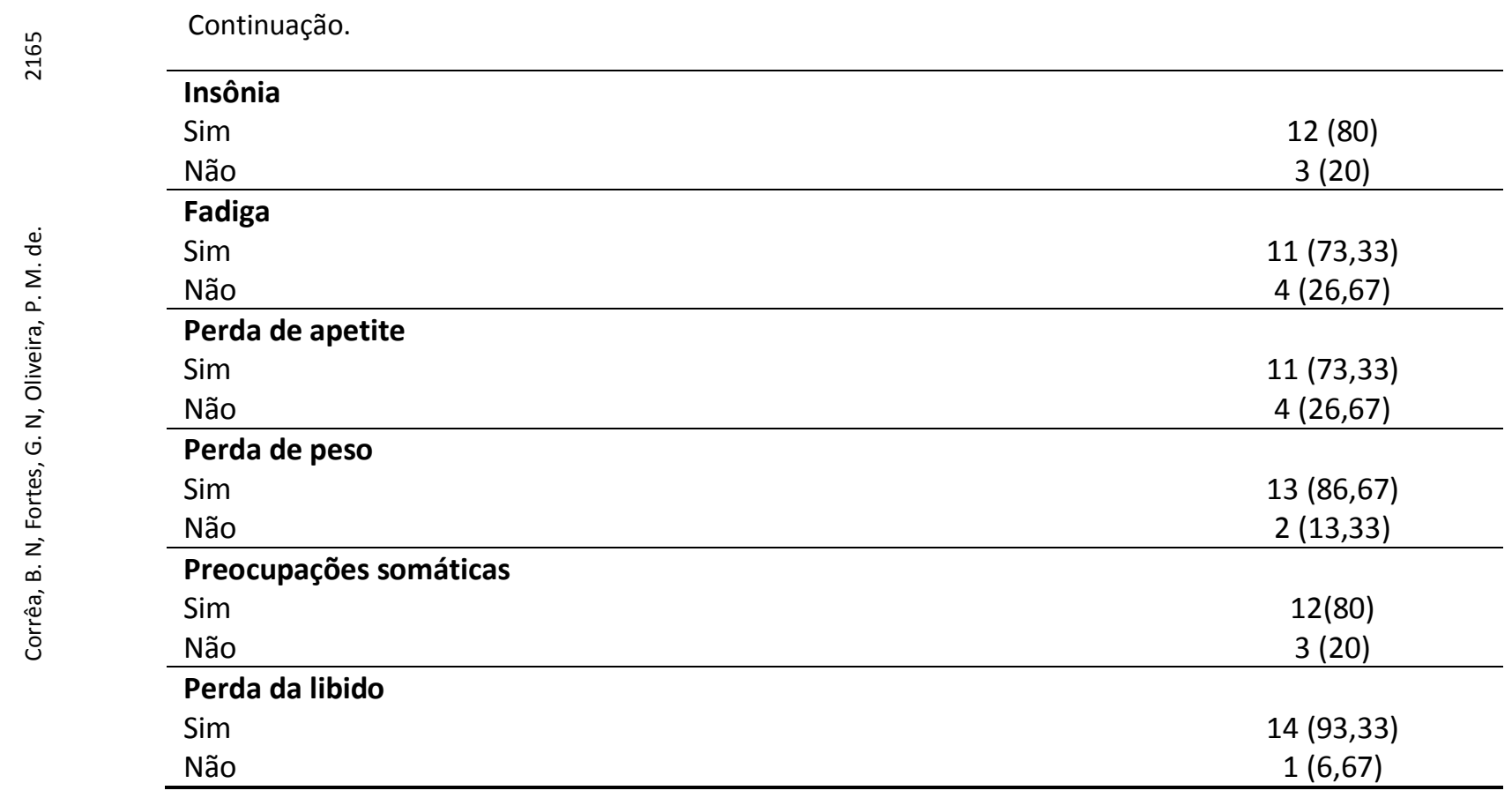

Fonte: Dados da pesquisa. 
No que se refere ao percentual quanto à intensidade dos itens de depressão, a perda de libido se destacou por representar $80 \%$ das respostas graves (bastante ou muito), seguida pela perda de peso $(53,33 \%)$, falta de apetite $(53,33 \%)$. Um estudo ${ }^{29}$ avaliou a qualidade de vida e prevalência de sintomas depressivos em pacientes oncológicos submetidos à radioterapia. A perda de libido seguido da perda de peso também foram os itens que receberam a maior porcentagem de respostas graves no BDI, corroborando com os resultados encontrados neste estudo. É importante destacar que nesta pesquisa, as mulheres identificadas com sinais e sintomas depressivos foram encaminhadas para atendimento médico psiquiátrico e psicológico para iniciar tratamento e acompanhamento de saúde mental.

A presença de uma doença debilitante, uso de medicações que provocam efeitos colaterais, a ocorrência de cirurgias e estados emocionais negativos, por vezes, concomitantes, podem justificar a alta prevalência da perda de libido em pacientes oncologicos ${ }^{22}$ e sintomas depressivos nesse público. Um estudo ${ }^{15}$ investigou a prevalência de incapacidades e aspectos associados para as mulheres com câncer do colo do útero, foram evidenciados a preocupação com o sistema reprodutivo $(96,6 \%)$, seguido de problemas nas funções da energia e do impulso $(70,3 \%)$, funções emocionais $(70,7 \%)$ e limitação para carregar e transportar objetos $(67,2 \%)$ foram as mais prevalentes. Observam-se possíveis repercussões na sexualidade feminina ${ }^{15}$ após diagnóstico e tratamento para CCU, além do simbolismo do útero ${ }^{15,30}$.

Autores destacam ser possível que o câncer cervical traga em si uma ideia dupla de morte- dela e da fertilidade - pelo significado construído historicamente e subjetivamente do útero como "geração da vida" em que a ameaça à possibilidade de ter filhos, no caso do útero, pode estar relacionado à ideia de punição ou fracasso na vida da mulher ${ }^{30}$. Dessa forma, é fundamental considerar a dimensão cultural do corpo, pois, a prática e o saber sobre o corpo não se encontram isentos de historicidade ${ }^{31}$.

Em um estudo ${ }^{32}$ sobre preditores de comprometimento cognitivo de pacientes recebendo tratamento curativo para câncer demonstrou que fatores estressantes do dia ainda podem levar as pacientes com CCU a abandonarem o tratamento, o que pode dificultar o acompanhamento assim como, a testagem psicológica, sendo importante, definir fatores preditivos para disfunções cognitivas relacionadas ao câncer. Faz-se importante, dessa forma, também considerar que o estado depressivo pode cursar com alterações cognitivas nos indivíduos ${ }^{33,34}$.

Os resultados do MEEM das participantes mostraram que a média de pontos obtidos, foi de $23,6( \pm 3,85)$ sendo que, a menor pontuação alcançada foi 15 e a maior, 30 pontos. A distribuição dos escores do MEEM das participantes apontou que $80 \%$ das mulheres apresentaram resposta normal e $20 \%$ das mulheres tiveram resposta alterada no MEEM, ou seja, não alcançaram o score mínimo, podendo ser indicativo de possível declínio cognitivo. As três participantes que apresentaram resposta alterada também mostraram, cada uma, estar em níveis de intensidade diferentes de depressão de leve, moderada e grave.

A literatura aponta que a depressão é uma síndrome que pode cursar com alterações cognitivas/déficit cognitivo, porém, ainda há controvérsias a respeito da gravidade ou magnitude desses déficits ${ }^{33}$. Uma das principais funções da cognição estudadas nos processos depressivos é a atenção, pela facilidade de avaliação da alteração que pode ser observada. Além disso, outro ponto importante é o desempenho do paciente com depressão referente ao seu nível de motivação, fator psicológico que interfere sobremaneira no funcionamento cognitivo e comportamental desses indivíduos ${ }^{35}$.

Uma revisão de literatura buscou a avaliação cognitiva de pacientes deprimidos, se destacaram que os domínios mais comumente afetados, são: atenção, memória (curta, longa, episódica, semântica, implícita, evocação, verbal e visual), velocidade de processamento, função executiva, flexibilidade mental, estruturação de estratégia e planejamento, processo de iniciação e supervisão, emoção e tomada de decisão ${ }^{33,34}$. Entretanto, é importante ressaltar que nem todos os pacientes deprimidos apresentam estes déficits, fato evidenciado neste estudo, e que pode ser justificado ao tamanho da amostra não ser significativa sem possibilidade de generalização, sendo necessários mais estudos com esse público.

Diante disso, a assistência a pessoas em tratamento oncológico requer além dos esforços curativos, o atendimento de demandas psicossociais, que possibilitem a visão holística a essas mulheres, onde a observação das situações e sintomas que permeiam a paciente no período 
de tratamento, possibilite prevenir possíveis quadros depressivos ${ }^{12,13,14}$.

$\mathrm{Na}$ detecção, prevenção e cuidado de adoecimentos junto ao paciente oncológico, devem ser analisados e resgatados o estilo de vida saudável (alimentação, atividades físicas, evitar o estresse, entre outros). Em nível secundário, abordados aspectos considerados de "cancerofobia", como sentimentos de medo com a possibilidade da descoberta de diagnóstico oncológico, buscando mediar à prevenção, produzindo também o deslizamento quanto ao mecanismo da negação (defesa) ou da evitação da realização de exames, como formas de não obter diagnóstico acerca da patologia. Em nível terciário, a prevenção aponta à relação das intervenções para facilitar o tratamento, para poder lidar com a aceitação e sofrimento resultantes do tratamento, tais como dor, náuseas, ansiedades, medo, depressão, e busca uma qualidade de vida mais adequado ao atual quadro que se encontra física e psicologicamente ${ }^{36}$.

Dessa forma, é fundamental que o manejo psicológico seja realizado com conhecimento teórico/técnico e ao mesmo tempo empático, através de uma escuta ativa e acolhedora. Pois, é pelo saber ouvir efetivamente que o psicólogo compreende qual o tipo de sofrimento que permeia a vivência da pessoa a partir das alterações emocionais que esteja apresentando $^{37}$. Destaca-se ainda que o psicólogo atuante na área de psicologia oncológica, pode prevenir e reduzir os sintomas emocionais e físicos causados pelo câncer e seus tratamentos, levando a pessoa a compreender o significado da experiência do adoecer, possibilitando assim, ressignificações desse processo e identificação e acompanhamento de possíveis aparecimentos de distúrbios psicopatológicos, como a depressão ${ }^{38}$. Aliados ao acolhimento psicológico, a psicoterapia de apoio, a entrevista de ajuda, atendimento em grupos com o paciente e família, além da possibilidade do atendimento pós-óbito para os familiares são ferramentas possíveis de intervenção de minimização de sofrimento ${ }^{39}$

\section{Considerações finais}

Considerando o impacto que o diagnóstico e tratamento do câncer de colo uterino podem acarretar na saúde da mulher, buscou-se analisar os níveis de depressão das mulheres em tratamento de câncer cervical no hospital de referência no Baixo Amazonas. Cabe ressaltar, que a maioria das participantes encontrava-se com idade acima dos 50 anos, ensino fundamental incompleto, com renda mensal inferior a um salário mínimo, em uma união estável e realizava radioterapia concomitante à quimioterapia.

O nível de depressão foi verificado por meio do Inventário de Depressão de Beck, o qual pôde identificar que os índices de depressão estavam presentes em $80 \%$ das mulheres em tratamento de câncer cervical em níveis leve a grave, com os seguintes sintomas mais recorrentes: diminuição da libido, perda de peso, preocupações somáticas, insônia, choro, dificuldades para trabalhar, fadiga e irritabilidade.

No que diz respeito ao Mini Exame do Estado Mental, 80\% das mulheres apresentaram resposta normal e $20 \%$ apresentaram resposta alterada, indicando possível declínio cognitivo, além disso, das participantes que apresentaram resposta alterada no exame mental, também em algum nível de depressão. Este estudo teve como fatores limitantes o número reduzido da amostra, o que impede a generalização dos resultados e o contexto de pandemia do Covid 19 que impactou a ida das participantes para realização do tratamento.

Diante do exposto, ressalta-se a importância da identificação dos índices e sintomas depressivos de forma regular e apropriada durante o período de tratamento para CCU, que considere a subjetividade das mulheres em adoecimento, bem como a relevância da identificação de fatores agravantes e de risco que possam contribuir para elevado sofrimento psíquico e emocional. Além disso, que sejam favorecidas ações educativas aliadas à presença de profissional da saúde mental e uma equipe multiprofissional que atue de maneira ética e profissional, disposta a acolher as queixas e desejos dos pacientes diante do adoecer e tratamento oncológico a fim de contribuir para melhoria da qualidade de vida.

\section{Referências}

1. Mendes CB, Nunes CR. Aspectos psicológicos dos pacientes com câncer de colo de útero, relacionado à prática radioterápica. Psicologia Revista. São Paulo; 2012; 21(1): 59-76.

2. BRASIL. Ministério da Saúde. Secretaria de Atenção a Saúde. Instituto Nacional de Câncer-INCA. ABC do câncer: abordagens básicas para o controle do câncer. (5). Rio de Janeiro (RJ): Ministério da Saúde; 2019. 
3. Ayres ARG, Silva GA. Prevalência de infecção do colo do útero pelo HPV no Brasil: revisão sistemática. Rev. Saúde Pública; 2010; 44 (5): 963-974.

4. FEBRASGO. Federacão Brasileira das Associacões de Ginecologia e Obstetrícia. Rastreio, diagnostico e tratamento do câncer de colo de útero. São Paulo (SP); 2017.

5. BRASIL. Instituto Nacional de Câncer José Alencar Gomes da Silva- INCA. Estimativa 2020: incidência de câncer no Brasil. Rio de Janeiro (RJ): Ministério da Saúde; 2019.

6. BRASIL. Instituto Nacional do Câncer. Estimativa 2018 - Incidência de Câncer no Brasil. Rio de Janeiro (RJ): Ministério da Saúde; 2017.

7. BRASIL. Ministério da Saúde. Diretrizes Brasileiras para o Rastreamento do Câncer do Colo do Útero: Brasília: Ministério da saúde; 2016; (2).

8. Tomazelli JG, Atty AT de M, Bertholasce ACA, Dias MBK. Tratamento Radioterápico no Sistema Único de Saúde: uma Análise do Período 2012 a 2016. Rev. Bras. Cancerol. 2018; 64(4): 461-9.

9. Fernandes WC, Kimura M. Qualidade de vida relacionada à saúde de mulheres com câncer de colo uterino. Revista Latino-americana de Enfermagem, Ribeirão Preto, 2010; 18 (3).

10. OMS. Organização Mundial da Saúde. Depression and Other Common Mental Disorders: Global Health Estimates. Geneva: World Health Organization; 2017.

11. APA. American Psychiatric Association. DSM5: Manual diagnóstico e estatístico de transtornos mentais. Porto Alegre: Artmed, 5 ed; 2014.

12. Almeida LHRB, Pereira YBAS, Oliveira TA. Radioterapia: percepção de mulheres com câncer cérvico-uterino. Rev. bras. enferm. 2008; 61 (4): 482-487.

13. Castro EK, Romeiro FB, Lima NB, Lawrenz P, Hass S. Percepção da doença, indicadores de ansiedade e depressão em mulheres com câncer. Psic., Saúde \& Doenças. 2015; 16( 3 ): 359-372.

14. Regino, P.A. Ansiedade, depressão e qualidade de vida de pacientes com câncer de mama e ginecológico frente aos efeitos da quimioterapia antineoplásica. Universidade Federal do Triângulo Mineiro, Uberaba, Minas Gerais, 2013.

15. Castaneda L, Bergmann A, Koifman R. Prevalência de incapacidades e aspectos associados em mulheres com câncer de colo do útero, Rio de Janeiro. Brasil. Cad. saúde colet., 2019; 27 (3): 307-315.
16. Moreira ECM et al. Estratégias psicoemocionais como mecanismos no enfrentamento do Câncer do Colo Uterino: revisão da literatura. Revista Científica Multidisciplinar Núcleo do Conhecimento. 2019; 12(06): 05-20.

17. Oliveira TMV. Amostragem não probabilística: adequação de situações para uso e limitações de amostras por conveniência, julgamento e cotas. Rev Adm On Line, 2001; 2(3).

18. OMS. Organização mundial da saúde. Folha informativa - COVID-19 (doença causada pelo novo coronavírus).

19. Brucki SMD, Nitrini R, Caramelli $P$, Bertolucci PHF, Okamoto IH. Sugestões para o uso do mini exame do estado mental no Brasil. Arq. Neuro-Psiquiatr. 2003; 61(3): 777-781.

20. Cunha JA. Manual da versão em português das Escalas de Beck. São Paulo. Casa do Psicólogo; 2001.

21. Conselho Nacional de Saúde (Brasil). Resolução n o 466, de 12 de dezembro de 2012. Brasília, 2012

22. Arrais TA. Qualidade de vida de mulheres usuárias do SUS com CCU, atendidas no hospital geral de Palmas-TO. Brasília: Universidade de Brasília, 2010; 64p.

23. Araújo CM, Aguiar VCM. Repercussões emocionais e comportamentais em mulheres com câncer no colo do útero. Revista Multidisciplinar Pey Këyo. 2017; 3(1):

24. Marques IA. Qualidade de Vida no Cancro do Colo do Útero: Um estudo com Mulheres da Amazônia em tratamento. Braga: Universidade do Minho, 2019; 35p.

25. Mascarello KC, Silva NF, Piske MT, Viana KCG, Zandonade E, Amorim MHC. Perfil Sociodemográfico e Clínico de Mulheres com Câncer do Colo do Útero Associado ao Estadiamento Inicial. Revista Brasileira de Cancerologia 2012; 58(3): 417-426.

26. Thuler LCS, Aguiar SS, Bergmann A. Determinantes do diagnóstico em estádio avançado do câncer do colo de útero no Brasil. Rev. Bras. Ginecol. e Obstet. 2014; 36(6):237-243.

27. Fisher D, Wedel, B. Anxiety and depression disoders in cancer patients: incidence, diagnosis and therapy. Magazine of European Medical Oncolology, 2012; (5) 1, 52-54.

28. Fagner $P$, Azevedo, R, Mauro M, Lima, D, Gaspar, K, Silva, V, Botega, N. Depressão e comportamento suicida em pacientes oncológicos: prevalência e fatores associados. Revista da Associação médica, 2010; 56(2):173-178. 
29. Pereira CAA, Passarin PN, Coimbra HJ, Pacheco GG, Rangel PM. Avaliação da Qualidade de Vida e Prevalência de Sintomas Depressivos em Pacientes Oncológicos Submetidos à Radioterapia. Rev. Bras. Cancerol, 2020;66(1):12775.

30. Melo, M; Barros E.N. Histerectomia e simbolismo do útero: possíveis repercussões na sexualidade feminina. Revista da SBPH, v.12, p.80-99, 2009.

31. Heilborn, ML. Articulando gênero, sexo e sexualidade: diferenças na saúde. In: Goldenberg, P, Marsiglia, RMG, Gomes, MHA. (Org.). O clássico e o novo: tendências objetos e abordagens em ciências sociais e saúde. Rio de Janeiro: Fiocruz, p. 197-208, 2003.

32. Lycke, M. et al. The distress thermometer predicts subjective, but not objective, cognitive complaints six months after treatment initiation in cancer patients. Journal Of Psychosocia Oncology, New York, v. 35, n. 6, p. 741-757, 2017.

33. Porto $P$, Hermolin $M$, Ventura $P$. Alterações neuropsicológicas associadas à depressão. Rev. bras. ter. comport. cogn.2002; 4( 1 ): 6370.

34. Rozenthal M, Laks J, Engelhardt, E.Aspectos neuropsicológicos da depressão. Revista de Psiquiatr. RS, 2004; 26(2): 204-212.

35. Laks, J, Marinho, VM, Rozenthal, $M$, Engelhardt, E. Neuropsicologia da Depressão. Revista Brasileira de Neurologia, p.97-102,1999.

36. BRASIL. Ministério da Saúde. Manual técnico de promoção da saúde e prevenção de riscos e doenças na saúde suplementar. Rio de Janeiro. ANS, 2009. 244.

37. Angerami - Camon VA, Vasconcellos EG , Gaspar KC, Carvalho HB C, Riechelmann JC, Sebastiani, RW. Psicologia da Saúde um novo significado para a prática clínica. São Paulo, Cengage Learning, 2014; (2).

38. Ferreira CRB, Schuh CA, Stein J, Tasca PC, Niquetti R. Atuação do psicólogo da saúde com pacientes oncológicos depressivos. Unoesc \& Ciência - ACBS; 2018; 9(1): 23-28.

39. Almeida RA, Malagris LEN. A prática da Psicologia da Saúde. Sociedade Brasileira de Psicologia Hospitalar. Rio de Janeiro, 2011; 14 (2).

40. Venâncio, JL. Importância da atuação do psicólogo no tratamento de mulheres com câncer de mama. Rio de Janeiro: Revista Brasileira de Cancerologia, 2004; 50(1); 55-63.

\section{Endereço para Correspondência}

\section{Brenda Nunes Corrêa}

Avenida Plácido de Castro, 1399- Aparecida, Santarém-Pará

Universidade do Estado do Pará

Cep: 68040-090

Email: brendanunespsi@gmail.com

Recebido em 28/03/2021

Aprovado em 01/05/2021

Publicado em 15/06/2021 